\title{
Characteristic dynamic rheological responses of nematic poly(p-phenylene terephthalamide) and cholesteric hydroxypropyl cellulose phases
}

\author{
Youngho Eom ${ }^{1}$, Dae Eon Jung ${ }^{1}$, Seung Sang Hwang ${ }^{2}$ and Byoung Chul Kim ${ }^{1}$
}

The liquid crystalline (LC) behavior of two lyotropic systems, poly(p-phenylene terephthalamide) (PPTA) solutions in sulfuric acid and hydroxypropyl cellulose (HPC) solutions in water, was compared on a dynamic rheological basis. The critical concentration (C*) curves showed much sharper transitions in PPTA solutions than in HPC solutions. The C* and saturation point (B-point) were observed at 7 and 10.5 wt\% for nematic PPTA solutions and at 40 and 53 wt $\%$ for cholesteric HPC solutions. Both lyotropic systems gave low yield stress $\left(\tau_{\mathrm{y}}\right)$ below $1 \mathrm{~Pa}$ at the B-point. With further increases in concentration, the $\tau_{\mathrm{y}}$ values of nematic PPTA and cholesteric HPC solutions were 461.48 and 6.03 , respectively. In the loss tangent (tan $\delta$ ) curves versus frequency, the tan $\delta$ of PPTA was monotonically decreased, but that of HPC was increased in the early stage of frequency, and this trend was more noticeable at higher concentrations. Two systems showed similar behaviors in molecular stress relaxation but different orientation relaxation time behaviors. With increased strain, the orientation relaxation time $\left(\lambda_{\mathrm{e}}\right)$ of the PPTA solutions was slightly increased, but that of the HPC solutions was decreased, and their $\lambda_{\mathrm{e}}$ values converged at $100 \%$ strain.

Polymer Journal (2016) 48, 869-874; doi:10.1038/pj.2016.46; published online 18 May 2016

\section{INTRODUCTION}

The liquid crystalline (LC) phase is an intermediate state between an ordered three-dimensional crystal and a disordered isotropic fluid. According to molecular ordering, the LC phases have been classified into nematic, cholesteric and smectic. In the nematic phase, the centers of gravity of the LC domains are randomly arranged, leading to little positional long range order. In the cholesteric phase, however, only orientational order exists, and the direction of the long axis of the molecules changes continuously within a specimen. ${ }^{1}$

Above the critical concentration $\left(\mathrm{C}^{\star}\right)$, the rigid chain polymer forms liquid crystals; in this process, the molecules with higher stiffness and aspect ratio form the anisotropic domain first. ${ }^{2,3}$ The LC polymer maintains its orientation during the fabrication and solidification step, owing to the long relaxation time of the ordered molecules. In particular, para-aromatic polyamides, well-known LC polymers, have been used in the production of organic fibers with high modulus and strength. ${ }^{4-8}$ In the spinning process of the LC polymer, it is important to rheologically control the domain morphology to obtain resultant products with the desired properties. Hence, it is crucial to understand the rheological responses to set up the optimized processing conditions.

The molecular arrangements of the nematic and cholesteric phases result in different material functions. Several publications on the rheology of lyotropic LC systems are available, but few papers addressed the differences in the rheological responses. ${ }^{9-18}$ This study investigated the characteristic features of the dynamic rheological responses of two lyotropic LC systems, poly( $p$-phenylene terephthalamide) (PPTA) in sulfuric acid $\left(\mathrm{H}_{2} \mathrm{SO}_{4}\right)$ and hydroxypropyl cellulose (HPC) in water, which are known to yield nematic and cholesteric phases, respectively.

\section{EXPERIMENTAL PROCEDURE}

Materials

The intrinsic viscosities of two LC polymers, PPTA and HPC, were $2.52 \mathrm{dl} \mathrm{g}^{-1}$ in $100 \% \mathrm{H}_{2} \mathrm{SO}_{4}$ and $0.99 \mathrm{dl} \mathrm{g}^{-1}$ in water, respectively. The viscosity-average molecular weights of the PPTA and HPC were calculated to be 16400 and $91100 \mathrm{~g} \mathrm{~mol}^{-1}$, respectively, by using the Mark-Houwink equation. ${ }^{19,20}$ PPTA was prepared by the polycondensation of terephthaloyl dichloride and p-phenylene diamine in dimethyl acetamide containing $\mathrm{LiCl}^{21}$ PPTA was dissolved in $100 \% \mathrm{H}_{2} \mathrm{SO}_{4}$ at $30^{\circ} \mathrm{C}$ for $2 \mathrm{~h}$, and was used immediately without aging. The commercial grade HPC was dissolved in an airtight ampoule to prevent evaporation of water during the long dissolution period. More than 3 months were needed to obtain a homogeneous solution of HPC.

Measurement of the rheological properties

The rheological properties were measured with a Rheometrics dynamic spectrometer RDS-7700 (Rheometrics, Inc., Union, NJ, USA) at $30^{\circ} \mathrm{C}$. In the RDS measurement, a parallel plate geometry was used. A quartz plate with a diameter of $50 \mathrm{~mm}$ was used to properly accommodate the torque exerted by the solutions. The solutions were stabilized for $30 \mathrm{~min}$ between the plates before measurement to relax the residual stress exerted during the gap setting.

${ }^{1}$ Department of Organic and Nano Engineering, Hanyang University, Seoul, Republic of Korea and ${ }^{2}$ Materials Architecting Research Center, Korea Institute of Science Technology, Seoul, Republic of Korea

Correspondence: Professor BC Kim, Department of Organic and Nano Engineering, Hanyang University, 222 Wangsimni-ro, Seoul, Seongdong-gu 04763, Republic of Korea. E-mail: bckim@hanyang.ac.kr

Received 9 December 2015; revised 29 February 2016; accepted 15 March 2016; published online 18 May 2016 

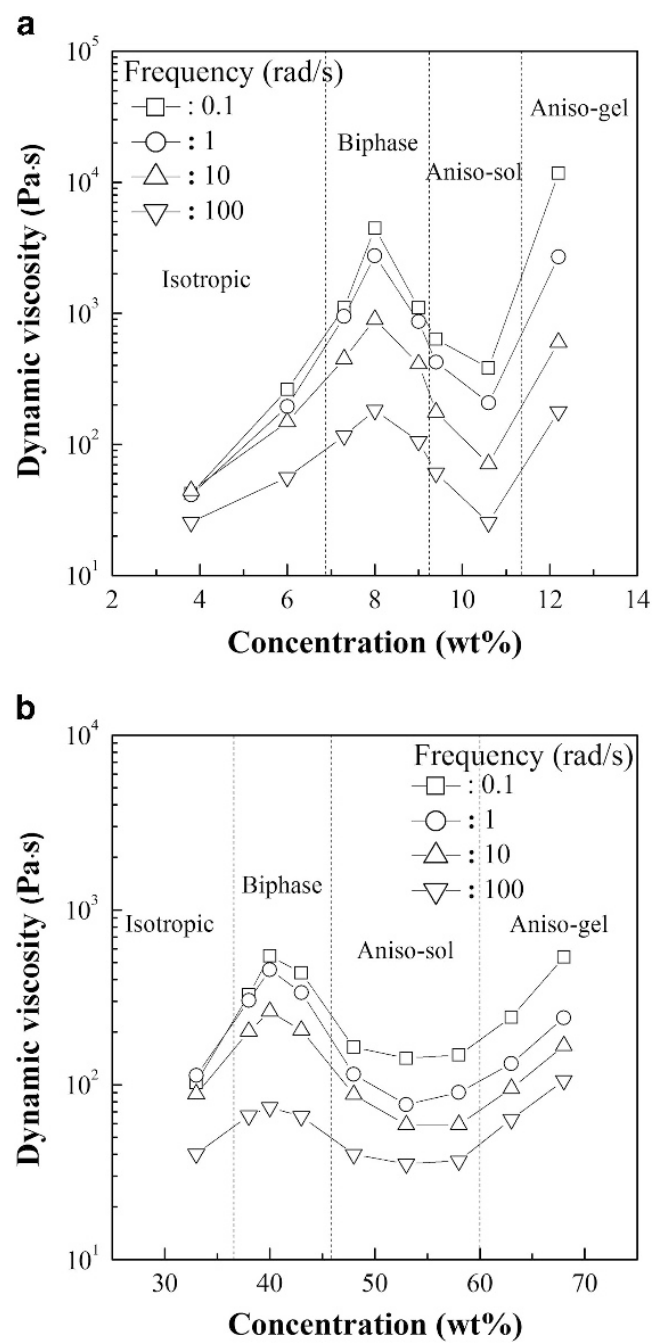

Figure 1 The critical concentration curves of (a) PPTA solutions in $\mathrm{H}_{2} \mathrm{SO}_{4}$ and (b) aqueous HPC solutions at $30^{\circ} \mathrm{C}$.

The relaxation modulus $\left(G_{\mathrm{r}}\right)$ was measured in the transient (step strain) mode of RDS. To determine the reproducibility of the results, all rheological measurements were repeated using the same procedure for PPTA and HPC specimens.

\section{RESULTS AND DISCUSSION}

Figures $1 \mathrm{a}$ and $\mathrm{b}$ show the variation of the dynamic viscosity $\left(\eta^{\prime}\right)$ with increase in concentration of PPTA solutions in $\mathrm{H}_{2} \mathrm{SO}_{4}$ and aqueous HPC solutions at $30^{\circ} \mathrm{C}$, respectively. Both lyotropic solutions yielded critical concentration $\left(\mathrm{C}^{\star}\right)$ curves typical of LC polymeric systems. The $\mathrm{C}^{\star}$ and saturation point (B-point) were observed at 8 and $10.5 \mathrm{wt} \%$ in PPTA solutions and at 40 and $53 \mathrm{wt} \%$ in HPC solutions, respectively. PPTA exhibited a sharper transition of the LC phase than HPC, indicating the rapid development of the nematic phase directly through the spontaneous orientation of the rigid rod-like PPTA chains. ${ }^{1}$ However, the gradual phase transition of the cholesteric phase resulted from the concentration-dependent conformation change of HPC chains, which produced rigidity. The semi-rigid HPC chains gradually transformed from coiled to stretched conformations as concentrations increased. This conformation change persisted over the entire concentration range, thus delaying the development of the LC phase. However, two lyotropic systems

exhibited clear $C^{\star}$ curves, even under high shear stress during the dynamic measurement. In fact, comparing the LC behavior between two lyotropic systems under steady shear has significant limitations because of the marked collapse of the LC domains.

The variation of the loss tangent $(\tan \delta)$ of the two solution systems with concentration at several frequencies is plotted in Figure 2. The $\tan \delta$ provides valuable information on the phase characteristics of the polymeric systems. ${ }^{22-24}$ An increase of tan $\delta$ indicates a trend toward a liquid-like character, but its decrease indicates a trend toward a solidlike character. PPTA solutions typically showed a reduction of $\tan \delta$ with increasing concentration. However, $\tan \delta$ increased as the concentration increased from $\mathrm{C}^{\star}$ to the B-point. The minimum value of $\tan \delta$ at $C^{\star}$ was ascribed to the three-dimensional network of randomly dispersed rigid PPTA chains. The development of the LC domains increased the liquid-like characteristics until the B-point. The large value of $\tan \delta$ at B-point indicated that the weak physical structure consisting of the LC domains was readily collapsed by shear stress via easy slippage between the domains. With a further increase of the concentration, the dense stacking of the domains in the aniso-gel region decreased $\tan \delta$. The cholesteric HPC showed the same tendency of $\tan \delta$ at $1 \mathrm{rads}^{-1}$ as nematic PPTA. However, the tan $\delta$ did not decrease with concentration, even after the B-point at 10 and 
$100 \mathrm{rad} \mathrm{s}^{-1}$. The cholesteric LC domains flowed very rapidly, even in the gel state, when a shear stress greater than $10 \mathrm{rad} \mathrm{s}^{-1}$ was applied.

The differences in the phase character were clearly ascertained by the $\tan \delta$ curves versus frequency in Figure 3. The PPTA solutions
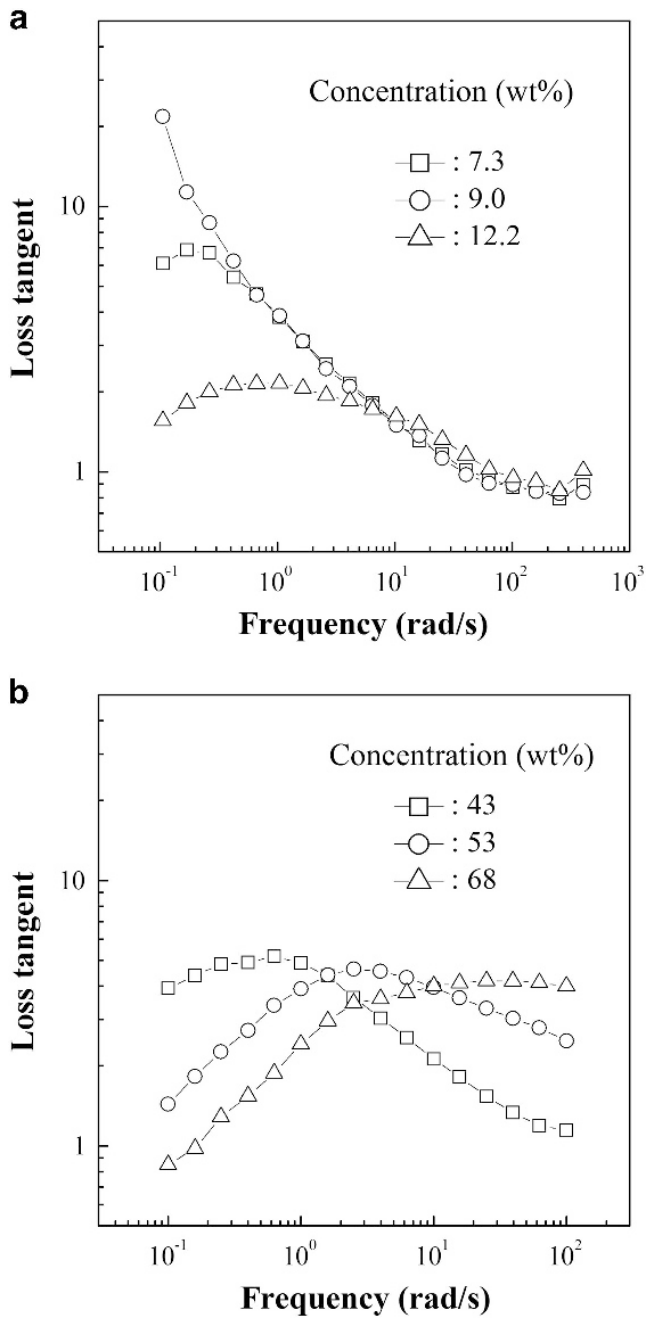

Figure 3 The plot of tan $\delta$ versus frequency for (a) PPTA solutions in $\mathrm{H}_{2} \mathrm{SO}_{4}$ and (b) aqueous $\mathrm{HPC}$ solutions at $30^{\circ} \mathrm{C}$. exhibited a monotonic decrease of $\tan \delta$ with an increase of frequency. However, the HPC solutions showed an increase of tan $\delta$ in the early stages of the test, which was more noticeable at higher concentrations. This increase indicated energy loss through phase change. Thus, external stress was damped through the continuous change of the cholesteric arrangement and the conformation of helicoidal HPC chains, as depicted in Scheme 1.

The strength of the physical structure in the solutions can be assessed by the yield stress $\left(\tau_{\mathrm{y}}\right)$, the minimum energy required to break

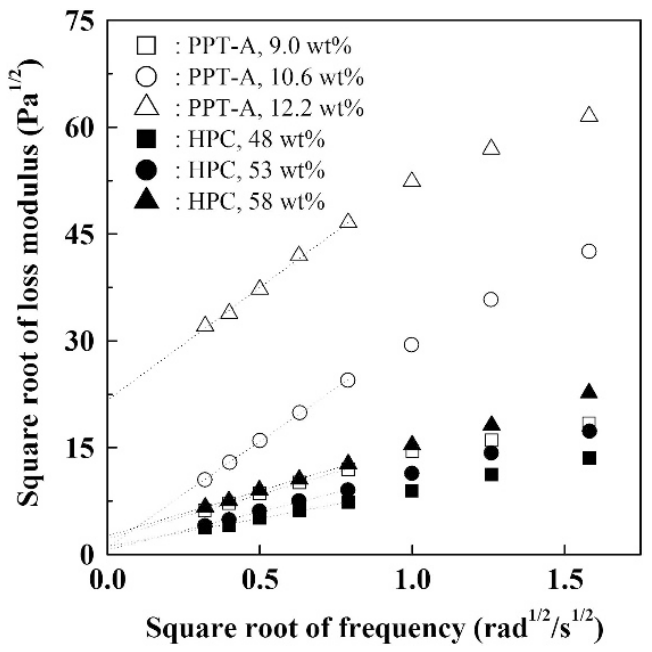

Figure 4 The Casson plot of PPTA solutions in $\mathrm{H}_{2} \mathrm{SO}_{4}$ and aqueous HPC solutions at several concentrations at $30^{\circ} \mathrm{C}$.

Table 1 The yield stress of the PPTA solutions in $\mathrm{H}_{2} \mathrm{SO}_{4}$ and aqueous HPC solutions at several concentrations at $30^{\circ} \mathrm{C}$

\begin{tabular}{lrrrr}
\hline PPTA in $\mathrm{H}_{2} \mathrm{SO}_{4}$ & & \multicolumn{3}{c}{ HPC in water } \\
\cline { 1 - 2 } \cline { 5 - 5 } Concentration (wt\%) & Yield stress (Pa) & & Concentration (wt\%) & Yield stress (Pa) \\
\hline 9.0 & 5.30 & & 48 & 1.27 \\
10.6 & 1.00 & & 53 & 0.42 \\
12.2 & 461.48 & 68 & 6.03 \\
\hline
\end{tabular}

Abbreviations: HPC, hydroxypropyl cellulose; PPTA, poly(p-phenylene terephthalamide)

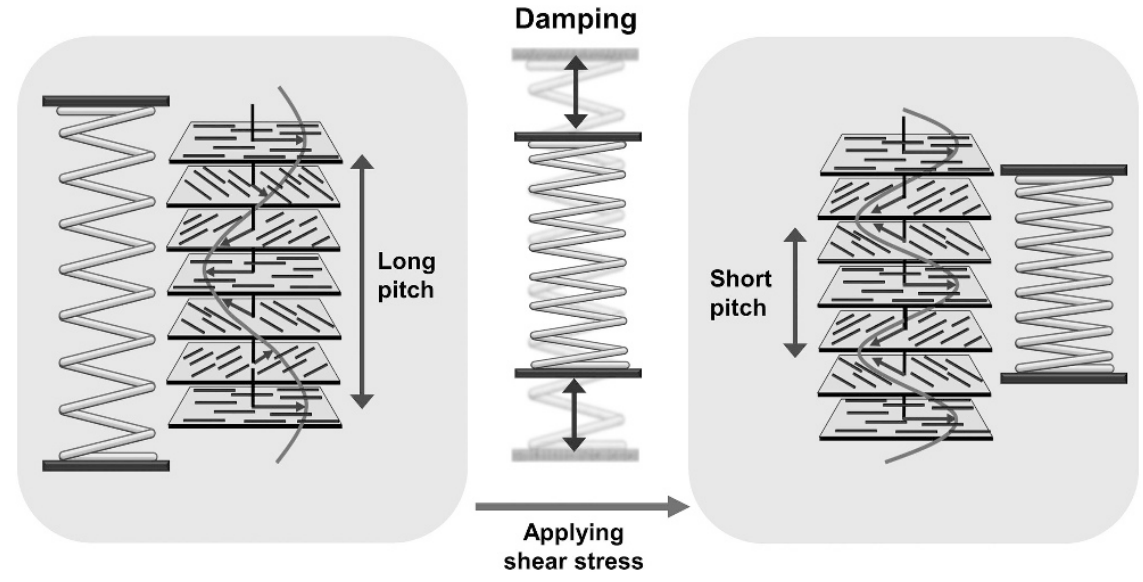

Scheme 1 The damping process of cholesteric HPC systems under shear stress. A full color version of this scheme is available at Polymer Journal online. 
down the structure. The $\tau_{\mathrm{y}}$, obtained from the Casson plot in Figure 4, at several concentrations above $\mathrm{C}^{*}$ is summarized in Table $1 .{ }^{25-27}$ As expected, both lyotropic systems produced low $\tau_{\mathrm{y}}$ in the aniso-sol region and B-point. However, the PPTA solution had a much larger value of $\tau_{\mathrm{y}}$ than the HPC solution in the aniso-gel region, 461.48 and 6.03 , respectively. This difference supports the $\tan \delta$ results, in that the gel structure of the cholesteric phase, unlike the nematic phase, was easily broken down by shear stress due to the damping process.

The heterogeneity of the solutions caused by the internal structure was further confirmed by a logarithmic plot of the storage modulus $\left(\mathrm{G}^{\prime}\right)$ versus loss modulus, the so-called Cole-Cole plot, as shown in Figure $5 .^{28-30}$ A slope of 2 on the Cole-Cole plot is empirically found with absolutely homogeneous polymer solutions without any physical structure. Thus, deviation from a slope of 2 is a measure of the heterogeneity of the polymer solution. The PPTA solutions showed a gradual decrease of the slope with concentration, which indicates that the extent of pseudostructure is closely related to the content of the LC domains. However, HPC solutions showed a sudden decrease in slope from 1.83 to 0.42 when the concentration exceeded $C^{\star}$, that is, the cholesteric HPC produced more heterogeneous phase than nematic PPTA in the anisotropic region. The HPC chains have been reported to release the strongly hydrogen-bonded water in the form of free water when they develop the LC domains. ${ }^{31}$ The sudden drop of the solubility of HPC in water is responsible for the deterioration of the homogeneity. The release process of the hydrogen-bonded water with HPC chains during the conformation change and the subsequent formation of the LC domain are illustrated in Scheme 2.

The LC polymeric systems exhibit two different relaxation modes for stress and orientation. The process of the stress relaxation on an individual molecular level is evaluated by tracing the relaxation time at the non-equilibrium state $\left(\lambda_{n}\right)$, and the relaxation process for the orientation in a collective molecular level can be accounted for by measuring the relaxation time to reach equilibrium $\left(\lambda_{\mathrm{e}}\right)$, which represents the recovery of the pseudostructure formed by the LC domains. First, $\lambda_{\mathrm{n}}$ can be calculated by Equation $(1)^{32}$

$$
J^{\prime}=\mathrm{G}^{\prime} /\left(\left[\eta^{*}\right] \omega\right)^{2}=\lambda /\left[\eta^{*}\right]
$$
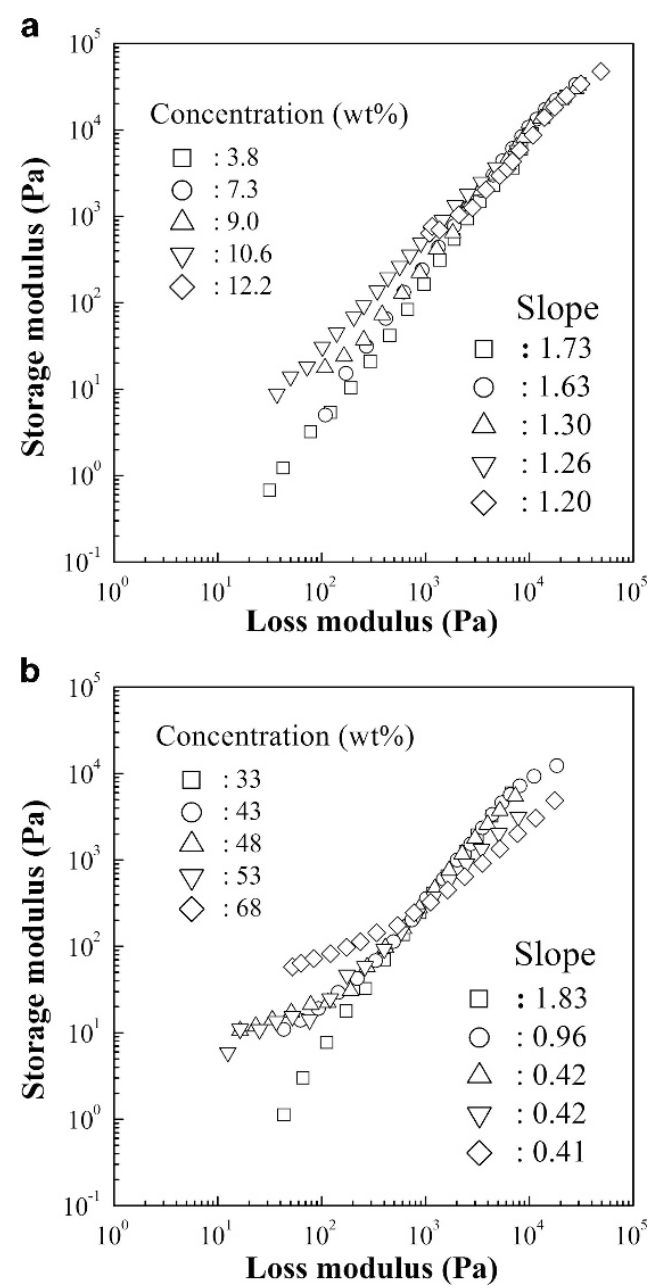

Figure 5 The modified Cole-Cole plot of (a) PPTA solutions in $\mathrm{H}_{2} \mathrm{SO}_{4}$ and (b) aqueous HPC solutions at several concentrations at $30^{\circ} \mathrm{C}$. The numbers on the graph are the slopes of the curves.

\section{Increase of concentration}

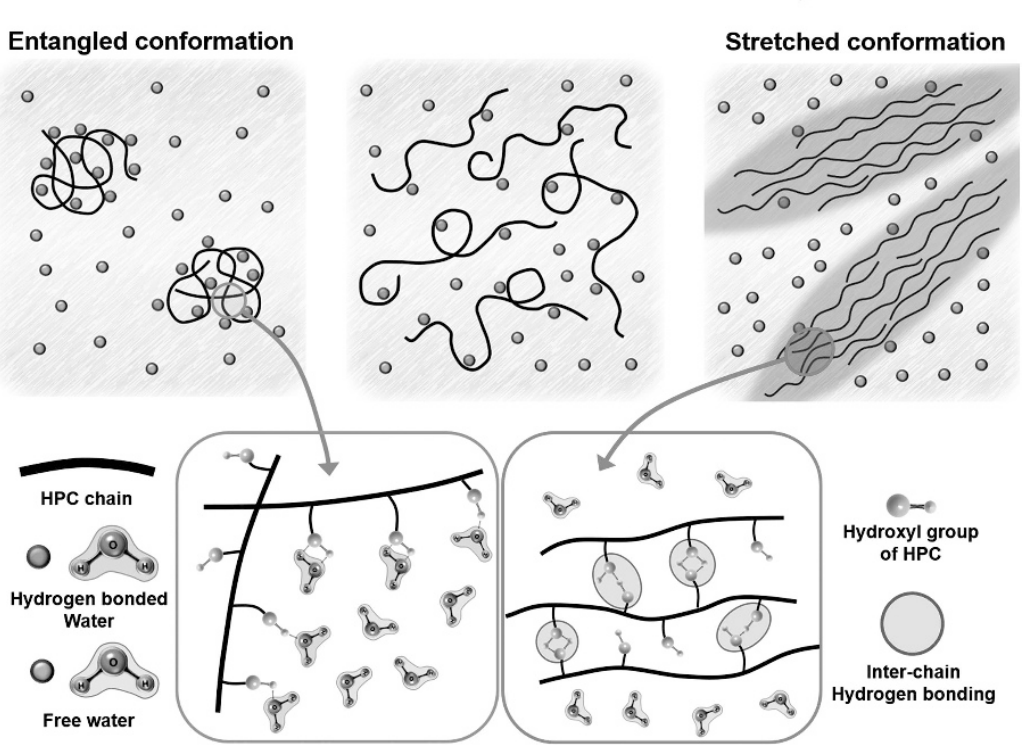

Scheme 2 The release process of strongly hydrogen-bonded water with HPC chains in the form of free water during the conformation change and subsequent LC domain development. A full color version of this scheme is available at Polymer Journal online. 


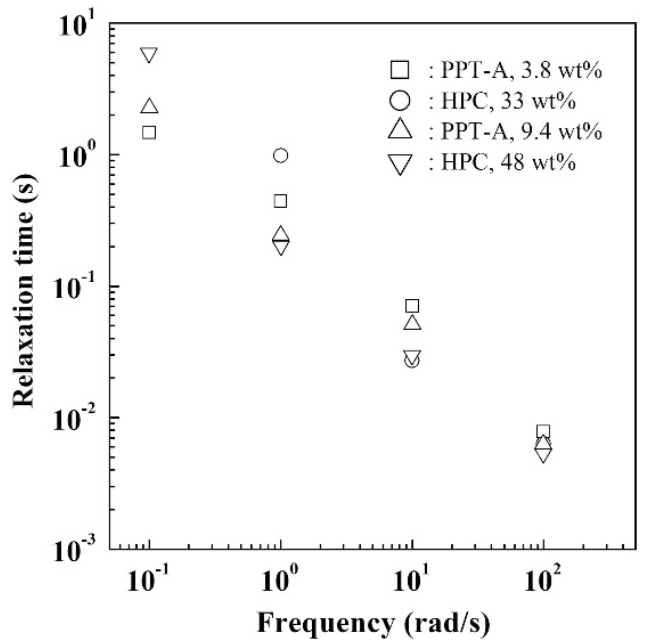

Figure 6 The variation of $\lambda_{n}$ with frequency for PPTA solutions in $\mathrm{H}_{2} \mathrm{SO}_{4}$ and aqueous HPC solutions at several concentrations at $30^{\circ} \mathrm{C}$.

in which $J^{\prime}$ and $\eta^{*}$ are the compliance and complex viscosity, respectively. The $\lambda_{\mathrm{n}}$ of the PPTA and HPC solutions is plotted in Figure 6. The two systems have similar $\lambda_{\mathrm{n}}$, in both the isotropic and anisotropic regions, whose values are simultaneously decreased with increasing frequency. The differences in the macroscopic LC structure between the nematic and cholesteric phases have little effect on the stress relaxation behavior of the individual molecules.

The $\lambda_{\mathrm{e}}$ and equilibrium modulus $\left(\mathrm{G}_{\mathrm{e}}\right)$ were obtained from the slope and intercept, respectively, in the plot of $G_{\mathrm{r}}$ against time in Figure 7, as suggested by Tobolsky and Murakami. ${ }^{33}$ The $G_{e}$, the strength of the pseudostructure formed by the LC domains, for the two solutions is presented in Figure 8. The HPC produced a greater $G_{e}$ than PPTA, thus suggesting a much higher content of the physical structure because the polymer concentration in the HPC solution was five times higher than that of the PPTA solution. However, the decrease of $G_{e}$ with strain was more noticeable in HPC than in PPTA, which further indicates that the physical structure of the HPC molecules is less tolerant to increases in shear stress.

In Figure 9, the HPC solutions yielded greater $\lambda_{\mathrm{e}}$ than the PPTA solutions, for the same reason underlying the trends in $G_{\mathrm{e}}$. However, the $\lambda_{\mathrm{e}}$ of the PPTA systems slightly increased but that of the HPC systems rapidly decreased with increased strain. In the case of the nematic system, applying shear stress breaks down the LC domains without changing the phase, and the $\lambda_{\mathrm{e}}$ increases with strain. However, the cholesteric phase has been reported to undergo a transition to the nematic phase with increasing shear stress. ${ }^{13}$ The convergence of the $\lambda_{\mathrm{e}}$ values of the two lyotropic systems indicates that the helicoidal structure of the HPC systems is transformed to a nematic-like directional order under very high shear.

\section{CONCLUSION}

The dynamic rheological properties of the lyotropic systems provided valuable information for comparing the phase characteristics between the nematic and cholesteric LC solutions. The differences in the responses were particularly noticeable at a high shear rate above $10 \mathrm{rad} \mathrm{s}^{-1}$. In addition, the solutions showed similar molecular behavior in the stress relaxation but markedly different behavior in the orientation relaxation. As a consequence, the lyotropic LC polymer
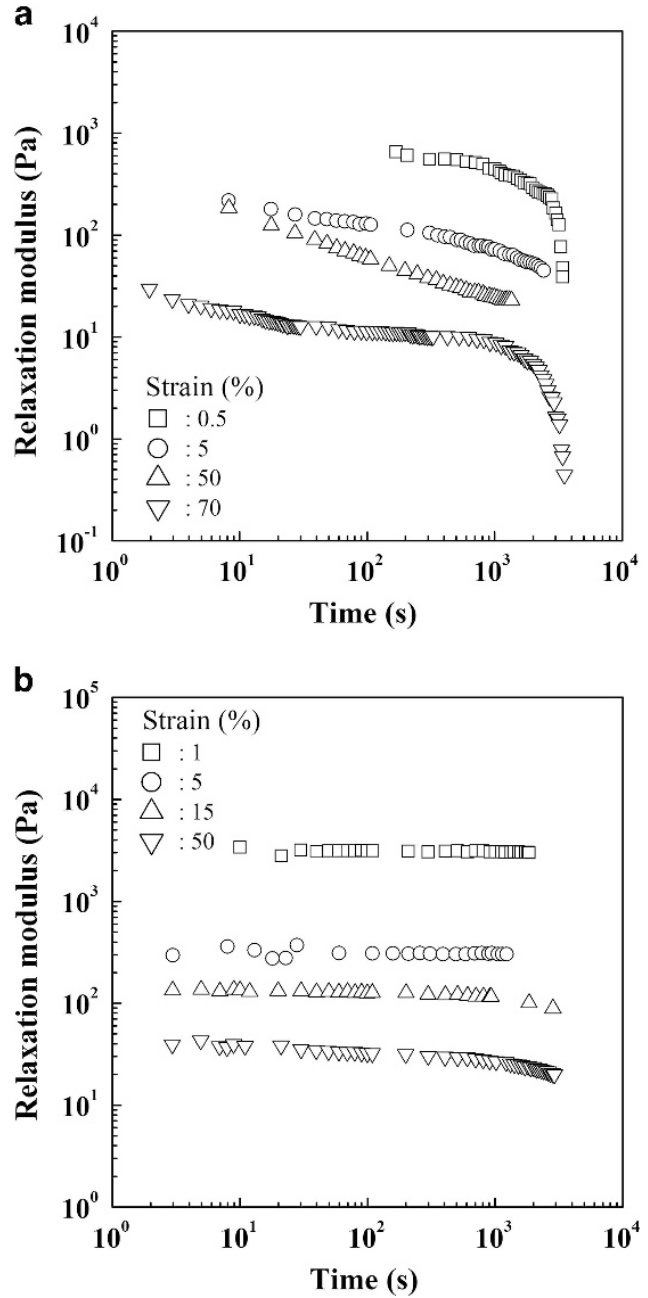

Figure 7 The variation of $G_{r}$ with time for (a) 9.4 wt $\%$ PPTA solution in $\mathrm{H}_{2} \mathrm{SO}_{4}$ and (b) $48 \mathrm{wt} \%$ aqueous HPC solutions at several strains at $30{ }^{\circ} \mathrm{C}$.

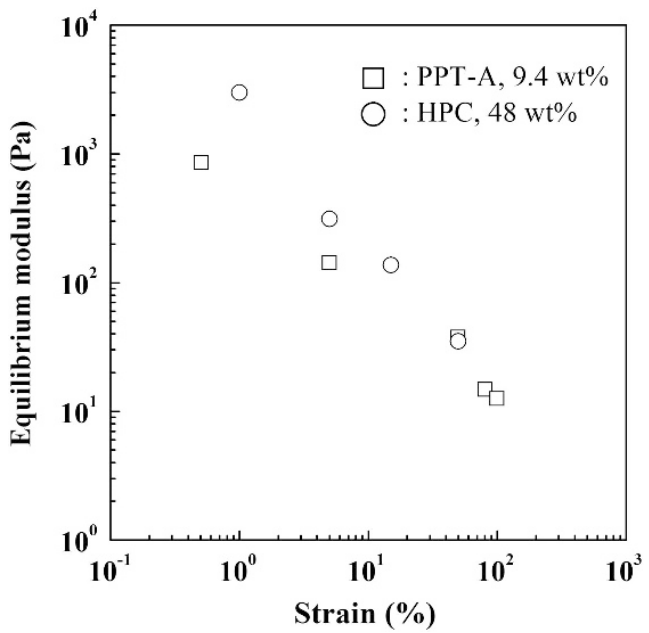

Figure 8 The variation of $G_{\mathrm{e}}$ with strain for $9.4 \mathrm{wt} \%$ PPTA solution in $\mathrm{H}_{2} \mathrm{SO}_{4}$ and $48 \mathrm{wt} \%$ aqueous $\mathrm{HPC}$ solution at $30^{\circ} \mathrm{C}$.

solutions with different ordering states should be addressed by using different processing conditions in the fabrication process to obtain products with the desired properties. 


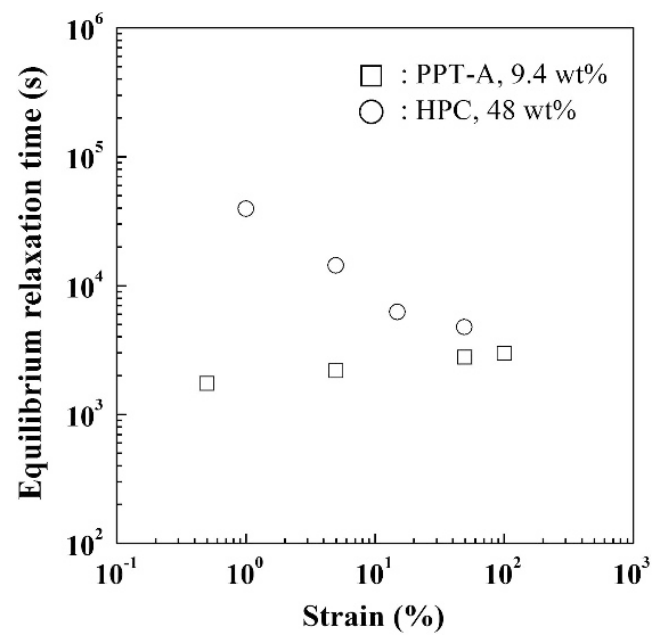

Figure 9 The variation of $\lambda_{\mathrm{e}}$ with strain for 9.4 wt\% PPTA solution in $\mathrm{H}_{2} \mathrm{SO}_{4}$ and 48 wt\% aqueous HPC solution at $30^{\circ} \mathrm{C}$.

\section{CONFLICT OF INTEREST}

The authors declare no conflict of interest.

1 Guido, S. Phase-behavior of aqueous-solutions of hydroxypropylcellulose. Macromolecules 28, 4530-4539 (1995).

2 Bair, T. I., Morgan, P. W. \& Killian, F. L. 'Poly(1,4-Phenyleneterephthalamides)polymerization and novel liquid-crystalline solutions. Macromolecules 10, 1396-1400 (1977).

3 Kwolek, S. L., Morgan, P. W., Schaefgen, J. R. \& Gulrich, L. W. Synthesis, anisotropic solutions, and fibers of poly(1,4-Benzamide). Macromolecules 10, 1390-1396 (1977).

4 Kotomin, S. V. Polymer molecular composites-new history. J. Thermoplast. Compos. Mater. 26, 91-108 (2013).

5 Naito, K. Tensile properties and weibull modulus of some high-performance polymeric fibers. J. Appl. Polym. Sci. 128, 1185-1192 (2013).

6 Preston, J. New high temperature polymers. I. Wholly aromatic ordered copolyamides. J. Polym. Sci. A Polym. Chem. 4, 529 (1966).

7 Preston, J., Smith, R. W. \& Sun, S. M. Fibers from aromatic copolyamides of limited order. Abst. Papers Am. Chem. Soc. 164, 1 (1972).

8 Yue, C. Y., Sui, G. X. \& Looi, H. C. Effects of heat treatment on the mechanical properties of Kevlar-29 fibre. Compos. Sci. Technol. 60, 421-427 (2000).

9 Baird, D. G. \& Smith, J. K. Dilute-solution properties of poly(1,4-Phenylene Terephthalamide) in sulfuric-acid. J. Polym. Sci. A Polym. Chem. 16, 61-70 (1978).

10 Fujii, S., Sasaki, N. \& Nakata, M. Rheological studies on the phase separation of hydroxypropylcellulose solution systems. J. Polym. Sci. B Polym. Phys. 39, 1976-1986 (2001).

11 Han, W. H., Zhao, T. Y. \& Wang, X. G. Dynamic viscoelastic relaxations of nematic solutions of poly(p-phenylene terephthamide) in sulfuric acid after cessation of shearing. Polymer 67, 72-79 (2015).
12 Hongladarom, K. \& Burghardt, W. R. Molecular orientation, 'Region I' shear thinning and the cholesteric phase in aqueous hydroxypropylcellulose under shear. Rheol. Acta 37, 46-53 (1998).

13 Hongladarom, K., Secakusuma, V. \& Burghardt, W. R. Relation between molecularorientation and rheology in lyotropic hydroxypropylcellulose solutions. J. Rheol. 38, 1505-1523 (1994).

14 Makarova, V. V., Tolstykh, M. Y., Picken, S. J., Mendes, E. \& Kulichikhin, V. G. Rheology-structure interrelationships of hydroxypropylcellulose liquid crystal solutions and their nanocomposites under flow. Macromolecules 46, 1144-1157 (2013).

15 Oertel, R. \& Kulicke, W. M. Viscoelastic properties of liquid-crystals of aqueous biopolymer solutions. Rheol. Acta 30, 140-150 (1991).

16 Regalado, E. J., Selb, J. \& Candau, F. Viscoelastic behavior of semidilute solutions of multisticker polymer chains. Macromolecules 32, 8580-8588 (1999).

17 Roschinski, C. \& Kulicke, W. M. Rheological characterization of aqueous hydroxypropyl cellulose solutions close to phase separation. Macromol. Chem. Phys. 201, 2031-2040 (2000)

18 Ying, Q. C. \& Chu, B. Characterization of Poly(1,4-Phenyleneterephthalamide) in concentrated sulfuric-acid. 4. Dilute and semidilute solution regimes. Macromolecules 19, 1580-1586 (1986).

19 Arpin, M. \& Strazielle, C. Characterization and conformation of aromatic polyamidesPoly(1,4-Phenylene Terephthalamide) and Poly(Para-Benzamide) in sulfuric-acid. Polymer 18, 591-598 (1977).

20 Clasen, C. \& Kulicke, W. M. Determination of viscoelastic and rheo-optical material functions of water-soluble cellulose derivatives. Prog. Polym. Sci. 26, 1839-1919 (2001).

21 Hong, S. I. \& Kim, B. C. A study on the engineering plastics(I)-factors affecting the molecular weight in the polycondensation of wholly aromatic polyamides. Polymer (Korea) 6, 336 (1982).

22 Chae, D. W., Kim, M. H. \& Kim, B. C. Temperature dependence of the rheological properties of poly(vinylidene fluoride)/dimethyl acetamide solutions and their electrospinning. Korea-Aust. Rheol. J. 22, 229-234 (2010).

23 Chae, D. W., Lim, J. H., Seo, J. S. \& Kim, B. C. Variation of physical properties of nylon$66 /$ clay nanocomposites with preparation conditions. Korea-Aust. Rheol. J. 24, 45-52 (2012).

24 Song, S. I. \& Kim, B. C. Characteristic rheological features of PVA solutions in water-containing solvents with different hydration states. Polymer 45, 2381-2386 (2004).

25 Bae, W. S., Kwon, O. J., Kim, B. C. \& Chae, D. W. Effects of multi-walled carbon nanotubes on rheological and physical properties of polyamide-based thermoplastic elastomers. Korea-Aust. Rheol. J. 24, 221-227 (2012).

26 Chae, D. W. \& Kim, B. C. Thermal and rheological properties of highly concentrated PET composites with ferrite nanoparticles. Compos. Sci. Technol. 67, 1348-1352 (2007).

27 Lyoo, W. S., Kim, J. H. Choi, J. H., Kim, B. C. \& Blackwell, J. Role of degree of saponification in the shear-induced molecular orientation of syndiotacticity-rich ultrahigh molecular weight poly(vinyl alcohol). Macromolecules 34, 3982-3987 (2001).

28 Chae, D. W. \& Kim, B. C. Effects of introducing silica particles on the rheological properties and crystallization behavior of poly(ethylene terephthalate). J. Mater. Sci. 42 , 1238-1244 (2007)

29 Chae, D. W., Kim, K. J. \& Kim, B. C. Effects of silicalite-1 nanoparticles on rheological and physical properties of HDPE. Polymer 47, 3609-3615 (2006).

30 Chae, D. W., Lee, K. H. \& Kim, Y. C. Rheological properties of ferrite nanocomposites based on nylon-66. J. Polym. Sci. B Polym. Phys. 44, 371-377 (2006).

31 Patra, A., Verma, P. K. \& Mitra, R. K. Slow relaxation dynamics of water in hydroxypropyl cellulose-water mixture traces its phase transition pathway: a spectroscopic investigation. J. Phys. Chem. B 116, 1508-1516 (2012).

32 Wissbrun, K. F. \& Griffin, A. C. Rheology of a thermotropic polyester in the nematic and isotropic states. J. Polym. Sci. B Polym. Phys. 20, 1835-1845 (1982).

33 Tobolsky, A. V. \& Murakami, K. Existence of a sharply defined maximum relaxation time for monodisperse polystyrene. J. Polym. Sci. 40, 443-456 (1959). 